\title{
Relations entre les structures géologiques, la composition chimique des eaux minérales et leurs orientations thérapeutiques
}

\author{
J. Canellas \\ Professeur émérite de Pharmacologie, Hydrologie à l'Université de Bordeaux II
}

\author{
B. Blavoux
}

Professeur d'Hydrogéologie à l'Université d'Avignon

\section{1 COMPOSITION CHIMIQUE DES EAUX MINÉRALES}

\subsection{Le problème de la classification chimique}

Grâce à la diversité de sa géologie, la France présente des eaux minérales de compositions et températures très variées. Certaines sont très minéralisées avec des résidus secs atteignant plusieurs grammes par litre (jusqu'à $300 \mathrm{~g} / \mathrm{l}$ à Salies du Salat), d'autres sont très peu minéralisées comme par exemple la source Charrier $(31 \mathrm{mg} / \mathrm{l})$ ou Volvic $(100 \mathrm{mg} / \mathrm{l})$. Certaines sont chaudes, voire très chaudes $\left(83{ }^{\circ} \mathrm{C}\right.$ à Chaudes-Aigues) alors que d'autres ont une température qui ne se distingue pas de celles des nappes phréatiques de leur environnement $\left(11,5^{\circ} \mathrm{C}\right.$ à Evian), si ce n'est par leur constance. Mais toutes ont en commun de présenter des propriétés favorables à la santé, dûment constatées par l'Académie Nationale de Médecine et dont la thérapeutique thermale peut tirer parti.

Toutefois, à la différence d'un médicament, il ne s’agit pas d'une molécule unique mais d'une solution complexe dont les propriétés thérapeutiques résultent vraisemblablement d'une pluralité de facteurs : concentrations en éléments majeurs, profils chimiques, présence d'éléments mineurs, d'éléments traces ou de gaz, température... C'est pourquoi la classification chimique, utilisée en hydrogéologie à partir des seuls éléments majeurs et considérant leurs concentrations absolues (Schoeller) et leurs concentrations relatives (Piper), reste insuffisante pour décrire l'eau minérale.

Si l'on se rapporte aux classifications habituellement utilisées en thermalisme on constate que trois grandes familles sont individualisées en tenant compte des élé-

Links between geological structures, the chemical composition of mineral waters and their therapeutic properties

The links between geology, the composition of mineral waters and their therapeutic properties are considered schematically having regard to an outline of the principal geological structures, the major characteristic elements in mineral waters and the therapeutic properties which enable treatment costs to be covered by Social Security.

This approach makes it possible to subdivide French mineral water resources into a number of major provinces based on geology, within which the water has a standard chemical profile and one or more specific therapeutic characteristics. However, small variations in chemical composition within a given province have resulted in other applications.

Finally, the central region of the country (the Massif Central) is taken as an example where there have been changes in therapeutic orientations, with the centre rich in sodium bicarbonate applied to diseases of the digestive system, as a result of the presence of chlorides and alkaline earths in the surrounding areas. 
ments majeurs que sont les anions hydrogénocarbonate (bicarbonate), sulfate et chlorure, lorsque ces éléments sont présents à des concentrations relativement importantes (>12 millimoles d'ions). On constitue souvent des sousgroupes à partir des cations majeurs en accolant à l'anion dominant le qualificatif de sodique, calcique, voire quelquefois magnésien. A partir de cette palette réduite existe un très grand nombre de variétés d'eaux minérales grâce à la présence d'éléments mineurs, d'éléments traces ou de gaz. A ces trois groupes, il faut ajouter les eaux sulfurées et les eaux oligométalliques ou pauciminéralisées. Ces dernières sont définies par leur faible minéralisation et des propriétés thérapeutiques qui les écartent du groupe possédant le même anion dominant.

\subsection{L'origine des éléments majeurs}

La minéralisation des eaux est fonction des éléments apportés à l'entrée du circuit hydrominéral par les précipitations et le sol, des éléments dissous en profondeur au contact de l'encaissant et éventuellement des éléments apportés des plus grandes profondeurs. La mise en solution est fonction de la température, de la pression et dans une moindre mesure de la vitesse de circulation de l'eau dans les zones de dissolution.

Les hydrogénocarbonates s'acquièrent communément par dissolution, d'une part du $\mathrm{CO}_{2}$ du sol produit par la minéralisation de la matière organique, d'autre part des carbonates du sol et des roches (calcaires, dolomies...). Dans ces conditions les hydrogénocarbonates ne peuvent guère dépasser des concentrations de 10 millimoles d'ions. Au-delà de ces valeurs on doit envisager la production de $\mathrm{CO}_{2}$ in situ par fermentation méthanique ou l'arrivée de $\mathrm{CO}_{2}$ profond magmatique ou métamorphique qui rendent l'eau à nouveau agressive vis-à-vis de son encaissant. Le terme carbogazeux s'applique évidemment aux sources présentant un dégagement de $\mathrm{CO}_{2}$ et par extension à ces eaux hyperminéralisées en bicarbonates.

Les eaux minérales tirent leur sulfate du lessivage des formations géologiques contenant du gypse et aussi de l'oxydation des sulfures, sulfures métalliques (pyrite) abondants dans certaines roches ou sulfures accompagnant le volcanisme. Dans le cas des évaporites, les concentrations en sulfates peuvent être très élevées et atteindre $1500 \mathrm{mg} / \mathrm{l}$. Elles s'accompagnent de teneurs très élevées en calcium. Les sulfures présents dans les eaux sulfurées sont le résultat d'une réduction biochimique des sulfates dissous.

Les chlorures proviennent du lessivage d'anciens dépôts évaporitiques contenant du sel gemme et sont alors associés au sodium jusqu'à des minéralisations totales de l'ordre de $300 \mathrm{~g} / \mathrm{l}$. Les chlorures en concentrations plus modestes peuvent avoir une origine magmatique. Ils sont présents dans les eaux minérales des régions à volcanisme récent mais aussi dans les eaux très profondes du socle cristallin.

A ces anions sont associés des cations majeurs.

Le sodium est fourni d'une manière générale par le lessivage de dépôts sédimentaires en milieu marin. Il résulte aussi de l'hydrolyse des minéraux silicatés tels que les plagioclases et peut être abondant dans les eaux carbogazeuses de socle cristallin.

Le calcium associé à l'ion sulfate est fourni par la dissolution du gypse. Il est aussi prédominant dans les eaux minérales circulant en milieu calcaire. Il peut exceptionnellement atteindre des teneurs élevées dans des eaux minérales carbogazeuses au contact de roches cristallines basiques.

Le magnésium est rarement le cation prédominant et peut provenir, soit des roches sédimentaires à partir de la mise en solution de la dolomie (carbonate double de calcium et de magnésium) ou celle d'évaporites, soit de roches cristallines ou métamorphiques par hydrolyse des minéraux silicatés ferromagnésiens (olivine, micas...) toujours exacerbée dans les eaux carbogazeuses.

Le potassium reste toujours un cation mineur et sa concentration ne devient significative que dans les eaux chlorurées très minéralisées en contact avec certaines évaporites.

\section{II — LES RELATIONS GÉOLOGIE/ COMPO- SITION CHIMIQUE}

\subsection{L'importance de la tectonique}

Le faciès chimique de l'eau minérale dépend de la nature des roches avec lesquelles elle est en contact et donc bien évidemment de la géologie. Par exemple, les eaux minérales qui tirent leur minéralisation du trias évaporitique sont très nombreuses en France.

Mais l'apparition des sources minérales et surtout thermales est due à l'existence d'un court-circuit hydraulique à forte perméabilité entre la profondeur et la surface résultant généralement d'une fracturation liée à un accident géologique récent. Les mécanismes qui permettent les circulations sont, obligatoirement, l'existence d'un gradient hydraulique important entre zone d'alimentation et zone d'émergence (relief accentué), accessoirement une élévation importante de température provoquant une baisse de densité de l'eau et/ou un dégagement abondant de $\mathrm{CO}_{2}$ mantellique (gaz-lift) à la faveur de failles profondes et ouvertes. De fait, les structures géologiques commandent l'apparition des sources minérales et si l'on examine la carte de France du thermalisme, on constate que la quasitotalité des stations se situe au sud-est d'une ligne Bordeaux-Sedan dans des régions affectées par une tectonique récente où l'on trouve les chaînes montagneuses des Pyrénées et des Alpes et les massifs anciens : partie du Massif Central à l'est du sillon houiller et Vosges bousculés par cette orogénèse alpine.

\subsection{Types d'eaux et provinces hydrominérales}

Nous avons essayé de rapprocher composition chimique des eaux minérales et grandes structures géologiques de notre pays. L'entreprise est difficile compte tenu de la diversité géologique de la France et du fait que les structures englobent des terrains de nature pétrographique très hétérogène. Ce rapprochement aboutit à la définition de types d'eaux issues des mêmes terrains, dans les mêmes 
STATIONS THERMALES FRANCAISES

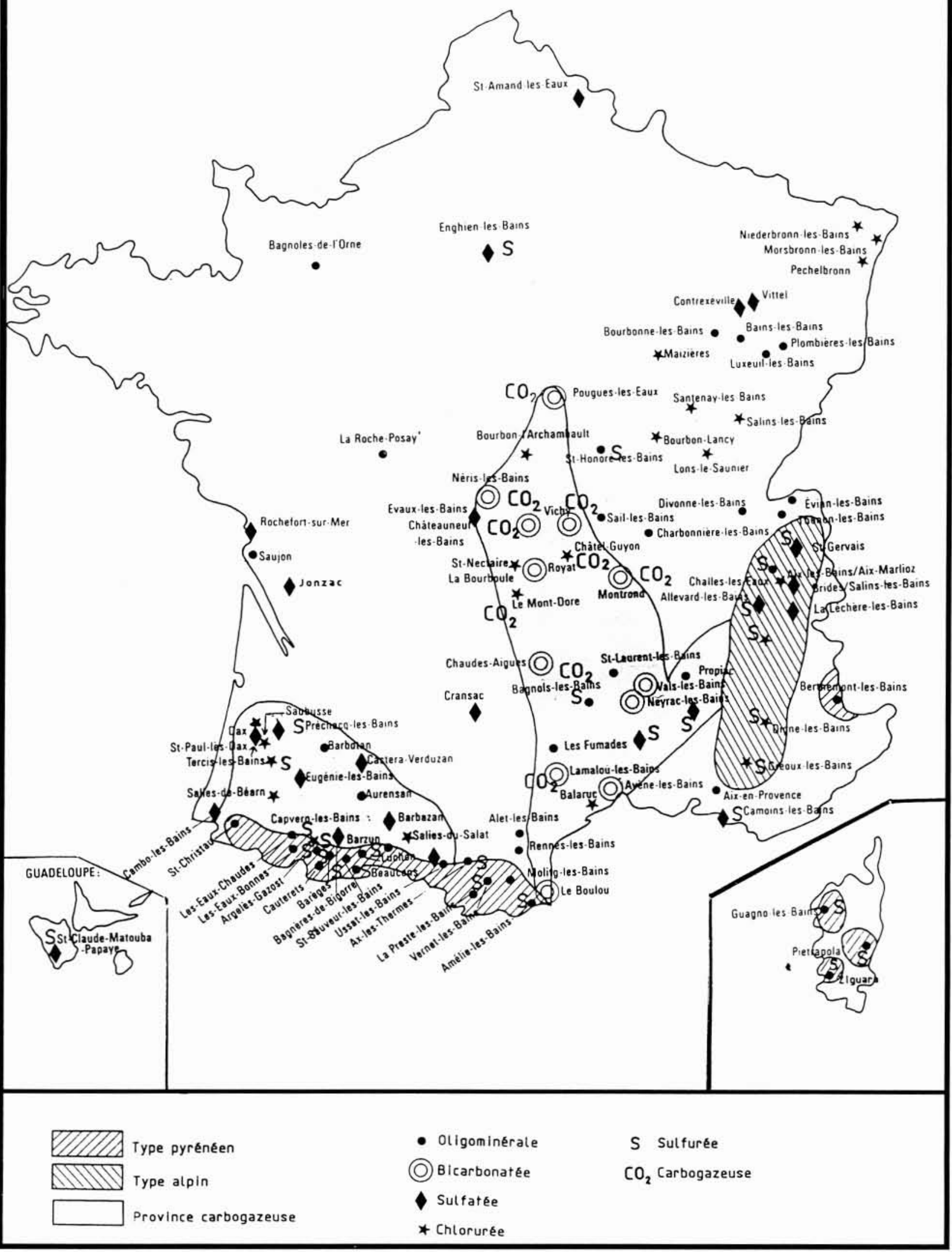


conditions structurales et hydrodynamiques et plus rarement à la délimitation de provinces hydrominérales homogènes.

2.2.1 Eaux sulfurées sodiques du type pyrénéen : peu minéralisées et chaudes avec un pH élevé, elles résultent d'un circuit profond et de longue durée dans les granites et gneiss des massifs montagneux. Elles sont caractéristiques de la chaîne primaire axiale des Pyrénées qui constitue une province hydrominérale homogène. Mais ce type d'eau se retrouve dans le massif du Mercantour au sud des Alpes et en Corse granitique.

2.2.2 Eaux sulfurées calciques du type alpin : plus minéralisées que les eaux du type précédent et chaudes, elles ont acquis des sulfates au contact des évaporites qui se retrouvent à la base et au front des grands chevauchements des Alpes occidentales. La réduction biochimique des sulfates leur confère le faciès sulfuré calcique caractéristique. Elles s'enrichissent parfois en chlorure de sodium et évoluent vers le type chloro-sulfaté mixte, ce qui fait que cette province hydrominérale des Alpes occidentales à la Provence est bien moins homogène que la précédente. Des sources sulfurées calciques froides se retrouvent hors de ce contexte à la faveur de circuits superficiels au contact d'évaporites oligocènes (Bassin Parisien, Languedoc, Provence).

2.2.3 Eaux sulfatées calciques et magnésiennes : leur apparition nécessite une double conjoncture: présence de terrains évaporitiques du Trias et existence d'une structure plissée ou faillée permettant la circulation et la remontée de l'eau minérale. Suivant la profondeur du circuit on distinguera les sources chaudes comme celles de la Province Alpine, quand elles ne sont pas affectées par la réduction des sulfates, celles de la Province Nord Pyrénéenne qui jalonne le chevauchement frontal nordpyrénéen, celles de la Province de Lorraine-Alsace de part et d'autre immédiate du massif cristallin des Vosges et les sources froides de la même province comme celles de Vittel et Contrexéville infiltrées dans les zones d'affleurement du Trias du Bassin Parisien.

2.2.4 Eaux chlorurées sodiques fortes: captées dans des stations thermales au nom évocateur (Salies, Salins), elles sont en contact intime avec le sel gemme au cours de leur circuit profond et se rencontrent dans la zone nordpyrénéenne à la faveur des diapirs de Trias salifère, dans le Jura externe où affleure le Trias salifère et en Savoie où l'on n'a toutefois jamais pu vérifier la présence de sel. Hormis le cas très particulier du Jura, on a du mal à distinguer une province hydrominérale chlorurée sodique au sein d'une province sulfatée calcique d'autant plus que toutes ces eaux liées aux dépôts évaporitiques présentent souvent un faciès mixte chloro-sulfaté calcique et sodique.

2.2.5 Eaux carbogazeuses de la Province périalpine : le caractère carbogazeux est acquis grâce à la remontée de $\mathrm{CO}_{2}$ magmatique par des accidents restés ouverts et affectant le socle cristallin. Les failles méridiennes des fossés d'effondrement du Massif Central et les grandes lignes de fractures de direction cévenole (NE-SW) qui ont joué en distension depuis le tertiaire, remplissent ces conditions. Elles délimitent la province des eaux bicarbonatées so- diques du Massif Central, stricto sensu. Toutefois cette province carbogazeuse du Massif Central fait partie d'une grande province carbogazeuse d'extension périalpine qui prend la France en écharpe du Roussillon jusqu'à l'Alsace. Elle intéresse des zones cristallines, l'extrémité orientale de la chaîne axiale pyrénéenne et les Vosges, avec des eaux au profil bicarbonaté sodique comme des zones à recouvrement sédimentaire, le Languedoc, la vallée du Rhône et les chaînes subalpines avec des eaux à profil bicarbonaté calcique. Elle pourrait inclure le front de la zone pennique des Alpes Occidentales dont le profil carbogazeux des eaux est masqué par le caractère chloro-sulfaté dominant.

Les grands accidents, par lesquels se dégage le $\mathrm{CO}_{2}$ profond, ont aussi permis les remontées de magmas volcaniques, plus limitées dans le temps et dans l'espace. C'est pourquoi dans le Massif Central, le profil chloruré sodique se superpose parfois au profil carbogazeux comme cela est évoqué dans les orientations thérapeutiques.

Le thermalisme des îles de la Martinique, de la Guadeloupe et de la Réunion est aussi et uniquement la conséquence d'un volcanisme récent.

2.2.6 Eaux oligométalliques : peu minéralisées, elles peuvent présenter tous les profils chimiques suivant la nature pétrographique de leur circuit. Elles se distinguent néanmoins de l'ensemble des sources banales de leur environnement par un ou plusieurs caractères originaux.

Chaudes, elles correspondent à des circuits profonds barrés par des grands accidents, par exemple à circulation fissurale dans les granites des Vosges (Plombières), à circulation fissurale et karstique dans les calcaires des chaînes jurassienne méridionale (Aix-les-Bains) ou provençale (Aix-en-Provence), à circulation en petit dans l'aquifère multicouche du Sud du Bassin d'Aquitaine où se différencie une province hydrominérale. Les eaux chaudes de l'aquifere profond des sables sous-molassiques (Eugénieles-Bains, Barbotan-les-Thermes) et des calcaires dolomitiques du Crétacé supérieur (Dax) y sont menées en surface par des structures à tectonique diapirique.

Froides, elles correspondent à des circuits exceptionnels, à débit conséquent, souvent réservées à l'embouteillage. Les sources minérales issues du Quaternaire lémanique (Evian, Thonon-les-Bains), d'une couche volcanique de la chaîne des Puys (Volvic), des calcaires urgoniens des Pyrénées (Ogeu-les-Bains) et du Jura (Divonne-les-Bains) illustrent la diversité de ces situations.

\section{LES RELATIONS GÉOLOGIE/ COMPO- SITION CHIMIQUE/ ORIENTATIONS THÉRAPEUTIQUES -}

\subsection{Les orientations thérapeutiques}

La prise en charge d'une cure thermale est assurée par la Sécurité Sociale à la condition que l'affection dont souffre le demandeur, entre dans le cadre de l'orientation "thérapeutique " attribuée à la station où se fera la cure. Les orientations thérapeutiques sont les suivantes :

- Voies respiratoires (VR); 
- Rhumatologie et séquelles de traumatismes ostéoarticulaires $(\mathrm{RH}) *$ :

- Neurologie (NEU) :

- Dermatologie (DER) ;

- Affections des muqueuses bucco-linguales, à l'exclusion des parodontopathies isolées; traitement des parodontopathies en complément d'une orientation thérapeutique autre que l'affection des muqueuses bucco-linguales (AMB) ;

- Thérapeutique des affections psychosomatiques (PSY) :

— Gynécologie et maladies de l'appareil génital (GYN) ;

- Phlébologie (PHL) :

- Maladies cardio-artérielles (MCA);

- Maladies de l'appareil digestif et maladies métaboliques $(\mathrm{AD})$;

- Maladies de l'appareil urinaire et maladies métaboliques $(\mathrm{AU})$ :

— Troubles du développement de l'enfant (TDE).

\subsection{Le cas général (Massif Central, Pyrénées, Alpes)}

Le Massif Central et sa production d'eaux riches en anhydride carbonique libre et dissous et en sodium vont justifier l'orientation maladies de l'appareil digestif. A cette orientation s'ajoutera celle des voies respiratoires avec des eaux contenant silice et arsenic.

La zone axiale des Pyrénées grâce aux eaux « sulfurées sodiques " possède les orientations : voies respiratoires, rhumatologie et séquelles de traumatismes ostéoarticulaires et dans un nombre limité de stations la dermatologie, la gynécologie et les maladies des voies urinaires.

Les terrains sédimentaires de la zone Nord-Pyrénéenne et des Alpes sont à l'origine d'eaux sulfatées, d'eaux chloro-sulfatées et d'eaux chlorurées :

- les orientations des eaux sulfatées calciques et magnésiennes froides concernent les maladies de l'appareil urinaire et de l'appareil digestif (ces eaux se rencontrent également dans les Vosges) :

- chaudes, les eaux sulfatées calciques intéressent la neurologie et la phlébologie, alors que les eaux chlorosulfatées gardent l'orientation phlébologie à laquelle s'ajoute dans ce cas la dermatologie.

Enfin, lorsqu'une partie des sulfates est réduite en sulfures (eaux « sulfurées calciques »), on a les orientations des eaux «sulfurées sodiques " (l'origine des « sulfures" est différente mais les orientations thérapeutiques sont les mêmes car on considère qu'une partie de l'activité est due aux diverses espèces réduites du soufre).

Les eaux chlorurées sodiques fortes se retrouvent au niveau de la zone Nord-Pyrénéenne avec leurs orientations spécifiques : Gynécologie et Maladies de l'appareil génital, Troubles du développement de l'enfant (ces eaux chlorurées sodiques se rencontrent également dans le Jura). Des eaux chlorurées sodiques moins minéralisées apparaissent à partir de terrains triasiques de Savoie.

(*) Les péloïdes (boues thermales), dont l'importance est grande dans le traitement des affections relevant de cette orientation, ne font pas l'objet de cet exposé.
Aux eaux oligométalliques chaudes de la partie sud du Bassin Aquitain (auxquelles s'ajoutent les boues thermales ou péloïdes) est attribuée l'orientation rhumatologie.

Le Massif Central possède, à côté de ses eaux bicarbonatées, des eaux oligométalliques issues de granites ou de terrains volcaniques qui font l'objet de l'embouteillage. Les eaux de ce groupe sont actives par leurs éléments traces dont certains sont des oligo-éléments, c'est-à-dire des éléments qui entrent dans la composition de molécules organiques nécessaires à des réactions fondamentales comme les enzymes ou à l'accomplissement d'une fonction particulière comme les hormones et les pigments.

Ces eaux se retrouvent dans toutes les orientations sauf les voies respiratoires et les troubles du développement de l'enfant.

\subsection{Le cas du Massif Central}

Le report sur une carte géologique simplifiée des principales orientations thérapeutiques permet à partir d'un centre « eau bicarbonatée sodique/maladies digestives » d'esquisser une zonalité thermale à partir de l'apparition en bordure de ce centre des chlorures et des alcalino-terreux qui entraîne de nouvelles indications alors que la thermalité est à l'origine de l'orientation rhumatologie devenue fréquente dans cette région.

En effet, l'exemple du Massif Central illustre la grande variété de ces eaux minérales dont la plupart, à côté de quelques eaux oligométalliques, sont bicarbonatées et qui vont voir apparaître à côté des hydrogénocarbonates des éléments mineurs susceptibles dans certains cas de devenir éléments majeurs et des éléments traces (Voir figure).

C'est ainsi que l'on trouvera les chlorures dans un vaste secteur en bordures Nord-Est, Nord, Nord-Ouest, Ouest, Sud-Ouest et Sud et les alcalino-terreux dans un secteur plus restreint en bordures Sud-Ouest, Sud, Sud-Est.

Les conséquences au niveau des orientations sont les suivantes :

- en bordures Sud-Ouest, Sud, Sud-Est, on trouvera les maladies de l'appareil digestif avec apparition du magnésium (Châtelguyon) ; les maladies cardio-artérielles avec les chlorures, le calcium et l'anhydride carbonique (Royat); les maladies de l'appareil urinaire avec la présence de chlorures (Saint-Nectaire) ; les maladies des voies respiratoires avec la silice et I'arsenic (La Bourboule, Le Mont-Dore) ;

- en bordures Ouest, Nord-Ouest, la neurologie est une orientation particulière qui apparaît avec la présence à côté des hydrogénocarbonates et chlorures (Néris). Ces caractéristiques se retrouvent avec l'orientation rhumatologie (Néris, Bourbon l'Archambault) ;

- en bordure Nord, on trouve une eau bicarbonatée calcique oubliée aujourd'hui mais bien connue autrefois pour l'importance de son embouteillage (Pougues);

- en bordures Nord, Nord-Est, les voies respiratoires avec la présence de sulfures et de chlorures (Saint-Honoré), la rhumatologie avec la présence de chlorures (BourbonLancy) ou de chlorures et de sulfates (Magnien, Santenay);

- en bordure Est, la dermatologie (Sail-les-Bains), la rhumatologie (Charbonnières) avec des eaux oligométalliques ; 


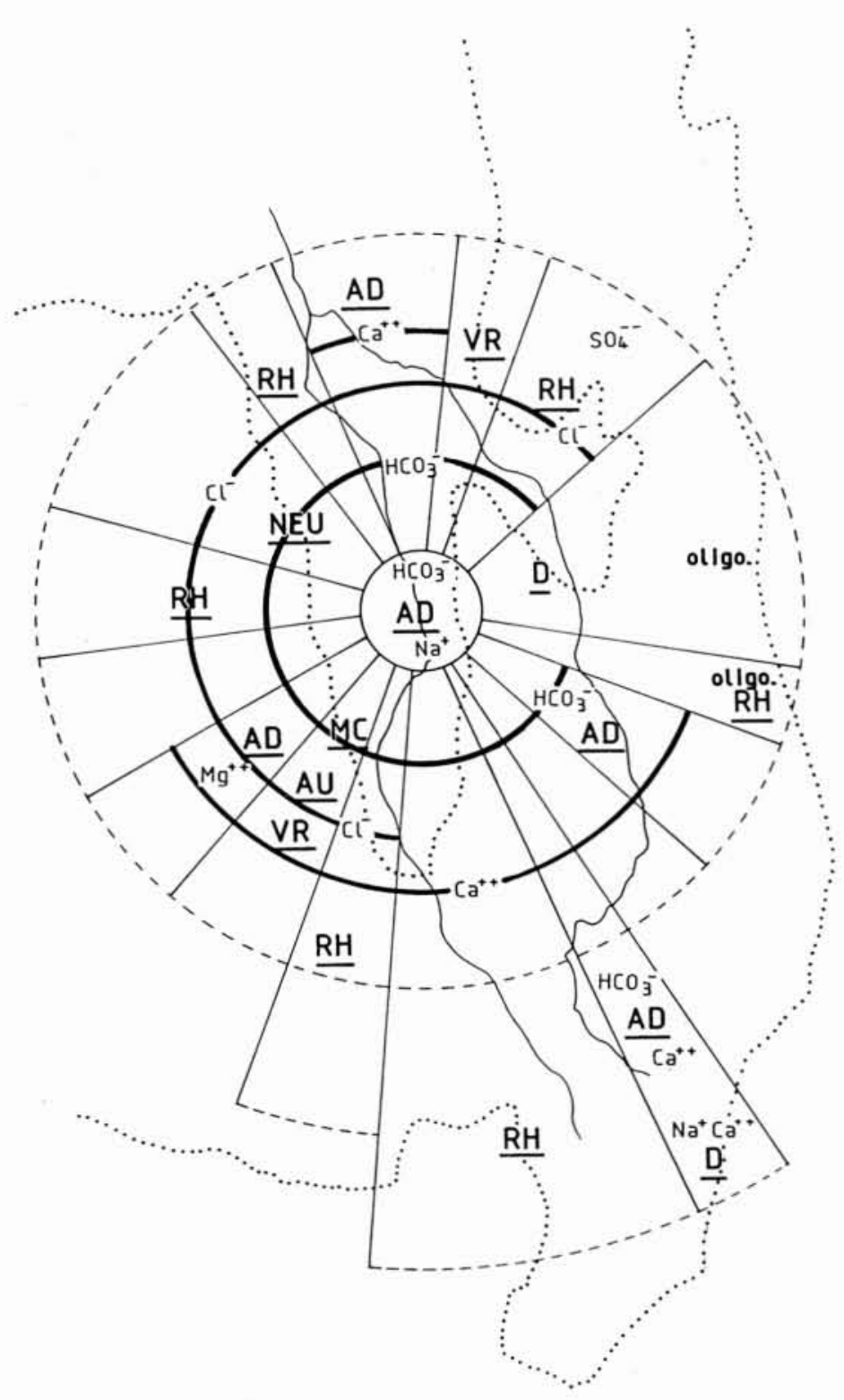

1. Eléments minéralisateurs et orientations thérapeutiques (Massif Central).

- en bordure Sud-Est, les maladies de l'appareil digestif (Saint-Galmier, Vals) et la dermatologie (Neyrac) avec « bicarbonate " et calcium ;

- enfin en bordure Sud, la rhumatologie (Bagnols, Chaudes-Aigues) avec chlorures et calcium ou (SaintLaurent-les-Bains) avec des eaux oligométalliques.

Ces quelques réflexions ont certes porté sur des données très globales : géologiques (histoire simplifiée du Massif Central), chimiques (seuls quelques ions sont retenus), thérapeutiques (une orientation regroupe diverses indications cliniques). Elles montrent cependant qu'à partir d'un anion et d'un cation dominants, les nuances apportées par les éléments mineurs, par les éléments traces (bien ou mal connus) et par les gaz peuvent être importantes.

\section{IV $\square$ CONCLUSION}

Les diverses considérations retenues mettent l'accent sur la multiplicité des facteurs qui font la spécificité des eaux minérales. Elles rappellent qu'il est souvent difficile de reconnaître les éléments pharmacologiquement actifs et justifient les indications différentes reconnues à des eaux d'origine et de composition apparemment voisines. 